PROCEEDINGS OF THE

AMERICAN MATHEMATICAL SOCIETY

Volume 140, Number 10, October 2012, Pages 3323-3331

S 0002-9939(2012)11177-3

Article electronically published on February 2, 2012

\title{
AN ANALOGUE OF HILBERT'S SYZYGY THEOREM FOR THE ALGEBRA OF ONE-SIDED INVERSES OF A POLYNOMIAL ALGEBRA
}

\author{
V. V. BAVULA \\ (Communicated by Birge Huisgen-Zimmermann)
}

\begin{abstract}
An analogue of Hilbert's Syzygy Theorem is proved for the algebra $\mathbb{S}_{n}(A)$ of one-sided inverses of the polynomial algebra $A\left[x_{1}, \ldots, x_{n}\right]$ over an arbitrary ring $A$ :

$$
\log \operatorname{gdim}\left(\mathbb{S}_{n}(A)\right)=\operatorname{l} \cdot \operatorname{gldim}(A)+n .
$$

The algebra $\mathbb{S}_{n}(A)$ is noncommutative, neither left nor right Noetherian and not a domain. The proof is based on a generalization of the Theorem of Kaplansky (on the projective dimension) obtained in the paper. As a consequence it is proved that for a left or right Noetherian algebra $A$ :
\end{abstract}

$$
\mathrm{w} \cdot \operatorname{dim}\left(\mathbb{S}_{n}(A)\right)=\mathrm{w} \cdot \operatorname{dim}(A)+n .
$$

\section{INTRODUCTION}

Throughout, ring means an associative ring with 1; module means a left module; $\mathbb{N}:=\{0,1, \ldots\}$ is the set of natural numbers.

Definition ([6]). Let $P_{n}(A)=A\left[x_{1}, \ldots, x_{n}\right]$ be a polynomial ring with coefficients in a ring $A$. The algebra $\mathbb{S}_{n}=\mathbb{S}_{n}(A)$ of one-sided inverses of $P_{n}(A)$ is a ring generated over the ring $A$ of by $2 n$ elements $x_{1}, \ldots, x_{n}, y_{n}, \ldots, y_{n}$ that satisfy the defining relations:

$$
y_{1} x_{1}=1, \ldots, y_{n} x_{n}=1, \quad\left[x_{i}, y_{j}\right]=\left[x_{i}, x_{j}\right]=\left[y_{i}, y_{j}\right]=0 \text { for all } i \neq j,
$$

where $[a, b]:=a b-b a$ is the commutator of elements $a$ and $b$.

By the very definition, the ring $\mathbb{S}_{n}$ is obtained from the polynomial ring $P_{n}$ by adding commuting, left (but not two-sided) inverses of its canonical generators. When $A=K$ is a field, the algebra $\mathbb{S}_{1}=K\langle x, y \mid y x=1\rangle$ is a well-known primitive algebra [17, p. 35, Example 2. Over the field $\mathbb{C}$ of complex numbers, the completion of the algebra $\mathbb{S}_{1}$ is the Toeplitz algebra which is the $C^{*}$-algebra generated by a unilateral shift on the Hilbert space $l^{2}(\mathbb{N})$ (note that $y=x^{*}$ ). The Toeplitz algebra is the universal $C^{*}$-algebra generated by a proper isometry. If char $(K)=0$, then the algebra $\mathbb{S}_{n}$ is isomorphic to the algebra $K\left\langle\frac{\partial}{\partial x_{1}}, \ldots, \frac{\partial}{\partial x_{n}}, \int_{1}, \ldots, \int_{n}\right\rangle$ of scalar integro-differential operators (via $x_{i} \mapsto \int_{i}, y_{i} \mapsto \frac{\partial}{\partial x_{i}}$ ).

Received by the editors June 2, 2010 and, in revised form, April 1, 2011.

2010 Mathematics Subject Classification. Primary 16E10, 16S85, 16S99.

Key words and phrases. The algebras of one-sided inverses, the algebra of one-sided inverses of a polynomial algebra, the global dimension, Hilbert's Syzygy Theorem, the projective dimension, the weak dimension.

(C)2012 American Mathematical Society Reverts to public domain 28 years from publication 
The algebra $\mathbb{S}_{n}=\mathbb{S}_{n}(K)$ was studied in detail in [6]: the Gelfand-Kirillov dimension of the algebra $\mathbb{S}_{n}$ is $2 n, \operatorname{cl} \cdot \operatorname{Kdim}\left(\mathbb{S}_{n}\right)=2 n$, and the weak and the global dimensions of $\mathbb{S}_{n}$ are $n$. The algebra $\mathbb{S}_{n}$ is neither left nor right Noetherian as was shown by Jacobson [16] when $n=1$ (see also Baer [2]). Moreover, it contains infinite direct sums of left and right ideals. In the series of three papers [7, 8] and [9], the group $G_{n}:=\operatorname{Aut}_{K-\operatorname{alg}}\left(\mathbb{S}_{n}\right)$ of automorphisms and the group $\mathbb{S}_{n}^{*}$ of units of the algebra $\mathbb{S}_{n}$ and their explicit generators were found (both groups are huge). The group $G_{1}$ was found by Gerritzen, 14). In 8 and [10] it is proved that $\mathrm{K}_{1}\left(\mathbb{S}_{1}\right) \simeq K^{*}$ and $\mathrm{K}_{1}\left(\mathbb{S}_{n}\right) \simeq K^{*}$ for all $n>1$ respectively.

Hilbert's Syzygy Theorem [13, [3] states that for any ring $A$,

$$
\operatorname{l.g} \operatorname{ldim}\left(A\left[x_{1}, \ldots, x_{n}\right]\right)=\operatorname{lggldim}(A)+n \text {. }
$$

The reason why it holds is the following Theorem of Kaplansky.

Theorem 1.1 (18). Let $A$ be a ring, $s$ be its regular and central element, $\bar{A}:=$ $A /(s)$. If $M$ is a nonzero $\bar{A}$-module with $\operatorname{pd}_{\bar{A}}(M)=n<\infty$, then $\operatorname{pd}_{A}(M)=n+1$.

The aim of the paper is to prove an analogue of Hilbert's Syzygy Theorem for the ring $\mathbb{S}_{n}(A)$.

Theorem 1.2. Let $A$ be a ring. Then

$$
\operatorname{l.gldim}\left(\mathbb{S}_{n}(A)\right)=\operatorname{l.gldim}(A)+n \text { and } \operatorname{r.gldim}\left(\mathbb{S}_{n}(A)\right)=\operatorname{r.gldim}(A)+n .
$$

The proof of this theorem is based on the following generalization of the Theorem of Kaplansky proved in the paper.

Theorem 1.3. Let $A$ be a ring, $s$ be a regular and left normal element of the ring $A, t$ be a right regular and right normal element of the ring $A$ such that $(s)=(t)$, and $\bar{A}:=A /(s)$. Suppose that either

(1) $t a-a t \in\left(t^{2}\right)$ for all elements $a \in A$ or

(2) the ring endomorphism $\overline{\tau_{t}}$ of the ring $\bar{A}$ is an automorphism (see (10).

If $M$ is a nonzero $\bar{A}$-module with $\operatorname{pd}_{\bar{A}}(M)=n<\infty$, then $\operatorname{pd}_{A}(M)=n+1$.

The Theorem of Kaplansky follows from Theorem 1.3 when we set $t=s$ (both conditions hold).

Analogues of Hilbert's Syzygy Theorems for some (mostly Noetherian) rings under additional assumptions on the coefficient ring $A$ (such as being Noetherian) were given in [1, [13, 44 and [5]. Generalizations of the Theorem of Kaplansky are the key in finding the global dimension of certain classes of Noetherian rings (such as skew polynomial rings, skew Laurent polynomial rings, rings of differential operators, etc.); see [11, [15, [19, [20] and [12].

\section{Proofs of Theorems 1.2 And 1.3}

At the beginning of the section we collect some basic facts on the ring $\mathbb{S}_{n}(A)$ and prove several lemmas that are used in the proofs of Theorems 1.2 and 1.3 .

The ring $\mathbb{S}_{n}(A)$ of one-sided inverses of a polynomial ring. Let $A$ be a ring. The ring $\mathbb{S}_{n}(A)$ is the direct sum of $\mathbb{N}^{2}$ copies of ${ }_{A} A$ and $A_{A}$,

$$
\mathbb{S}_{n}(A)=A \otimes_{\mathbb{Z}} \mathbb{S}_{n}(\mathbb{Z})=\bigoplus_{\alpha, \beta \in \mathbb{N}^{n}} A x^{\alpha} y^{\beta}=\bigoplus_{\alpha, \beta \in \mathbb{N}^{n}} x^{\alpha} y^{\beta} A,
$$


where $x^{\alpha}:=x_{1}^{\alpha_{1}} \cdots x_{n}^{\alpha_{n}}, \alpha=\left(\alpha_{1}, \ldots, \alpha_{n}\right), y^{\beta}:=y_{1}^{\beta_{1}} \cdots y_{n}^{\beta_{n}}$ and $\beta=\left(\beta_{1}, \ldots, \beta_{n}\right)$. The $\operatorname{ring} \mathbb{S}_{n}(A)$ contains the ideal

$F(A):=\bigoplus_{\alpha, \beta \in \mathbb{N}^{n}} A E_{\alpha \beta}$, where $E_{\alpha \beta}:=\prod_{i=1}^{n} E_{\alpha_{i} \beta_{i}}(i), \quad E_{\alpha_{i} \beta_{i}}(i):=x_{i}^{\alpha_{i}} y_{i}^{\beta_{i}}-x_{i}^{\alpha_{i}+1} y_{i}^{\beta_{i}+1}$.

Note that $E_{\alpha \beta} E_{\gamma \rho}=\delta_{\beta \gamma} E_{\alpha \rho}$ for all elements $\alpha, \beta, \gamma, \rho \in \mathbb{N}^{n}$, where $\delta_{\beta \gamma}$ is the Kronecker delta function. Let $\mathfrak{a}_{n}(A)$ be the ideal of the algebra $\mathbb{S}_{n}(A)$ generated by the elements $E_{00}(i), i=1, \ldots, n$ (if $n=1$, then $\mathfrak{a}_{1}(A)=F(A)$ ). The factor ring $\mathbb{S}_{n}(A) / \mathfrak{a}_{n}(A)$ is canonically isomorphic to the Laurent polynomial $A$-algebra $L_{n}(A):=A\left[x_{1}, x_{1}^{-1}, \ldots, x_{n}, x_{n}^{-1}\right]$ via the $A$-isomorphism:

$$
\mathbb{S}_{n}(A) / \mathfrak{a}_{n}(A) \rightarrow L_{n}(A), \quad x_{i} \mapsto x_{i}, \quad y_{i} \mapsto x_{i}^{-1}, \quad i=1, \ldots, n,
$$

since $y_{i} x_{i}=1$ and $1-x_{i} y_{i}=E_{00}(i) \in \mathfrak{a}_{n}(A)$.

Suppose that the ring $A$ admits an involution $*$; i.e., it is an anti-isomorphism $\left((a b)^{*}=b^{*} a^{*}\right.$ for all elements $\left.a, b \in A\right)$ with $a^{* *}=a$ for all elements $a \in A$. The involution $*$ can be extended to the involution $*$ on the ring $\mathbb{S}_{n}(A)$ by the rule

$$
x_{i}^{*}=y_{i}, \quad y_{i}^{*}=x_{i}, \quad i=1, \ldots, n .
$$

In particular when $A$ is a commutative ring and $*$ is the identity map on $A$ we have the $A$-involution (i.e. $a^{*}=a$ for all $a \in A$ ) on the ring $\mathbb{S}_{n}(A)$. There is a split ring $A$-epimorphism

$$
\pi: \mathbb{S}_{n}(A) \rightarrow A, \quad x_{i} \mapsto 1, \quad y_{i} \mapsto 1, \quad i=1, \ldots, n
$$

Therefore,

$$
\mathbb{S}_{n}(A)=A \oplus\left(x_{1}-1, \ldots, x_{n}-1, y_{1}-1, \ldots, y_{n}-1\right) .
$$

Lemma 2.1(2) shows that the ideal $\left(x_{1}-1, \ldots, x_{n}-1, y_{1}-1, \ldots, y_{n}-1\right)$ of the ring $\mathbb{S}_{n}(A)$ is equal to the ideal $\left(x_{1}-1, \ldots, x_{n}-1\right)$ or $\left(y_{1}-1, \ldots, y_{n}-1\right)$ or $\left(t_{1}-1, \ldots, t_{n}-1\right)$, where $t_{i}=x_{i}, y_{i}$.

The polynomial $\operatorname{ring} P_{n}(A)=A\left[x_{1}, \ldots, x_{n}\right]$ is an $\mathbb{S}_{n}(A)$-module. In more detail,

$$
\mathbb{S}_{n}(A) P_{n}(A) \simeq \mathbb{S}_{n}(A) /\left(\sum_{i=0}^{n} \mathbb{S}_{n}(A) y_{i}\right)=\bigoplus_{\alpha \in \mathbb{N}^{n}} A x^{\alpha} \overline{1}, \quad \overline{1}:=1+\sum_{i=1}^{n} \mathbb{S}_{n}(A) y_{i},
$$

and the action of the canonical generators of the $A$-algebra $\mathbb{S}_{n}(A)$ on the polynomial ring $P_{n}(A)$ is given by the rule:

$$
x_{i} * x^{\alpha}=x^{\alpha+e_{i}}, \quad y_{i} * x^{\alpha}=\left\{\begin{array}{ll}
x^{\alpha-e_{i}} & \text { if } \alpha_{i}>0, \\
0 & \text { if } \alpha_{i}=0,
\end{array} \text { and } E_{\beta \gamma} * x^{\alpha}=\delta_{\gamma \alpha} x^{\beta},\right.
$$

where the set $e_{1}:=(1,0, \ldots, 0), \ldots, e_{n}:=(0, \ldots, 0,1)$ is the canonical basis for the free $\mathbb{Z}$-module $\mathbb{Z}^{n}=\bigoplus_{i=1}^{n} \mathbb{Z} e_{i}$.

An element $r$ of a ring $R$ is called a left normal if $R r=(r)$, i.e. $r R \subseteq R r$, where $(r)=\operatorname{Rr} R$ is the (two-sided) ideal of the $\operatorname{ring} R$ generated by the element $r$. An element $r$ of a ring $R$ is called a right normal if $r R=(r)$, i.e. $R r \subseteq r R$. An element $r$ of a ring $R$ is called a normal if it is left and right normal, equivalently, $R r=r R$ (since $R r=(r)=r R$ ).

Lemma 2.1. Let $A$ be a ring. Then:

(1) The element $x-1 \in \mathbb{S}_{1}(A)$ is left normal and the element $y-1 \in \mathbb{S}_{1}(A)$ is right normal; i.e. $\mathbb{S}_{1}(A)(x-1)=(x-1)$ and $(y-1) \mathbb{S}_{1}(A)=(y-1)$. 
(2) $(x-1)=(y-1),{ }_{A} \mathbb{S}_{1}(A)_{A}=A \bigoplus(x-1)$, and $F(A) \subseteq(x-1)$.

Remark. Lemma 2.2 shows that the element $x-1$ is not a right normal element and the element $y-1$ is not a left regular element of $\mathbb{S}_{n}(A)$.

Proof. By (11) and since $x-1 \in \mathbb{S}_{1}(\mathbb{Z})$ and $y-1 \in \mathbb{S}_{1}(\mathbb{Z})$, it suffices to prove the lemma for $A=\mathbb{Z}$. The $\mathbb{Z}$-algebra $\mathbb{S}_{1}=\mathbb{S}_{1}(\mathbb{Z})$ is generated by the elements $x$ and $y$ over the ring $\mathbb{Z}$. The left ideal $\mathbb{S}_{1}(x-1)$ is an ideal of the ring $\mathbb{S}_{1}$ since

$$
(x-1) y=(1-(x-1) y)(x-1) .
$$

In more detail, using the equalities $E_{00}=1-x y$ and $E_{00} x=0$, we see that

$$
(1-(x-1) y)(x-1)=\left(E_{00}+y\right)(x-1)=-E_{00}+1-y=x y-1+1-y=(x-1) y \text {. }
$$

By (므), the left ideal $\mathbb{S}_{1}(x-1)$ is an ideal of $\mathbb{S}_{1}$, i.e. $x-1$ is a left normal element of the ring $\mathbb{S}_{1}$. The ring $\mathbb{S}_{1}$ admits the involution $*$ over the ring $\mathbb{Z}: y^{*}=x$. Then the element $y-1=(x-1)^{*}$ is right normal. Notice that

$$
1-y=y(x-1) \in(x-1) .
$$

Then $(y-1) \subseteq(x-1)$ and the opposite inclusion follows when we apply the involution $*$ to the original one, i.e. $(x-1)=(y-1)$. Now,

$$
\mathbb{S}_{1}=\mathbb{Z}[x] \otimes_{\mathbb{Z}} \mathbb{Z}[y]=(\mathbb{Z} \oplus \mathbb{Z}[x](x-1)) \otimes_{\mathbb{Z}}(\mathbb{Z} \oplus \mathbb{Z}[y](y-1))=\mathbb{Z} \oplus(x-1)
$$

since the $\mathbb{Z}$-algebra epimorphism $\pi: \mathbb{S}_{1} \rightarrow \mathbb{Z}, x \mapsto 1, y \mapsto 1$, is a split epimorphism. Since $\operatorname{ker}(\pi)=(x-1)$ and $\pi\left(E_{i j}\right)=0$ for all elements $i, j \in \mathbb{N}$, we have the inclusion $F \subseteq(x-1)$.

Lemma 2.2. Let $A$ be a ring. Then neither the right ideal $(x-1) \mathbb{S}_{1}(A)$ nor the left ideal $\mathbb{S}_{1}(A)(y-1)$ is an ideal of the ring $\mathbb{S}_{1}(A)$.

Proof. By (11) and since $x-1, y-1 \in \mathbb{S}_{1}(\mathbb{Z})$, we may assume that $A=\mathbb{Z}$. In view of the involution $*$ on the ring $\mathbb{S}_{1}=\mathbb{S}_{1}(\mathbb{Z})$ it suffices to show that the right ideal $(x-1) \mathbb{S}_{1}$ is not an ideal of the ring $\mathbb{S}_{1}$ since $\mathbb{S}_{1}(y-1)=\left((x-1) \mathbb{S}_{1}\right)^{*}$. Supposing that $(x-1) \mathbb{S}_{1}$ is an ideal of the ring $\mathbb{S}_{1}$, we seek a contradiction. Since $F(\mathbb{Q})=\mathbb{Q} \otimes_{\mathbb{Z}} F($ where $F=F(\mathbb{Z}))$ is the least nonzero ideal of the algebra $\mathbb{S}_{1}(\mathbb{Q})$ (Proposition 2.5(2), [6]) and $\mathbb{S}_{1} \subseteq \mathbb{S}_{1}(\mathbb{Q})$, it follows from (2) that $F(\mathbb{Q}) \cap(x-$ $1) \mathbb{S}_{1}=m F$ for a nonzero integer $m$ since $F(\mathbb{Q}) \subseteq(x-1) \mathbb{S}_{1}(\mathbb{Q})$. Since $m E_{00} \in$ $F(\mathbb{Q}) \cap(x-1) \mathbb{S}_{1}$ there exists an element $a \in \mathbb{S}_{1}$ such that $(x-1) a=m E_{00}$. Taking this equality modulo $F$ and using the fact that the factor ring $\mathbb{S}_{1} / F$ is a Laurent polynomial algebra $\mathbb{Z}\left[x, x^{-1}\right]$ over $\mathbb{Z}$ which is a domain, we see that $a \in F$. The ideal $F$ is the direct sum $\bigoplus_{j \in \mathbb{N}} E_{\mathbb{N}, j}$ of left ideals $E_{\mathbb{N}, j}=\bigoplus_{i \in \mathbb{N}} \mathbb{Z} E_{i j} \simeq \mathbb{S}_{1} \mathbb{Z}[x]$ $\left(E_{0 j} \mapsto 1\right)$. Recall that $E_{i j}=x^{i} y^{j}-x^{i+1} y^{j+1}$. Since the left multiplication by the element $x-1$ in $\mathbb{Z}[x]$ is an injection and $E_{00} \in E_{\mathbb{N}, 0}$, we must have $a \in E_{\mathbb{N}, 0}$. Notice that ${ }_{\mathbb{Z}[x]} E_{\mathbb{N}, 0} \simeq \mathbb{Z}[x] \mathbb{Z}[x], E_{00} \mapsto 1$. The equality $(x-1) a=m E_{00}$ implies that $m \in(x-1) \mathbb{Z}[x]$, a contradiction.

An element $r$ of a ring $R$ is called left regular if $s r=0$ implies $s=0$ for $s \in R$. Similarly, right regular is defined, and regular means both left and right regular.

Lemma 2.3. Let $A$ be a ring. The elements $x-1$ and $y-1$ of the algebra $\mathbb{S}_{1}(A)$ are regular. 
Proof. By (11) and since $x-1, y-1 \in \mathbb{S}_{1}(\mathbb{Z})$, we may assume that $A=\mathbb{Z}$. In view of the involution $*$ on the algebra $\mathbb{S}_{1}=\mathbb{S}_{1}(\mathbb{Z})\left(y^{*}=x\right)$, it suffices to show that the element $x-1$ is regular. Suppose that $l(x-1)=0$ and $(x-1) r=0$ for some elements $l, r \in \mathbb{S}_{1}$. We have to show that $l=r=0$. Since the element $x-1$ is a regular element of the factor ring $\mathbb{S}_{1} / F=\mathbb{Z}\left[x, x^{-1}\right]$, we see that $l, r \in F$. We have seen in the proof of Lemma 2.2 that ${ }_{\mathbb{Z}[x]} F \simeq \mathbb{Z}[x]^{(\mathbb{N})}$; hence $r=0$. The ideal $F$ is the direct sum $\bigoplus_{i \in \mathbb{N}} E_{i, \mathbb{N}}$ of right ideals $E_{i, \mathbb{N}}=\bigoplus_{j \in \mathbb{N}} \mathbb{Z} E_{i j} \simeq \mathbb{S}_{1} / x \mathbb{S}_{1} \simeq \mathbb{Z}[y]_{\mathbb{S}_{1}}$ $\left(E_{i 0} \mapsto 1\right)$. In the right $\mathbb{S}_{1}$-module $\mathbb{Z}[y], y^{i} x=\left\{\begin{array}{ll}y^{i-1} & \text { if } i>0, \\ 0 & \text { if } i=0 ;\end{array}\right.$ i.e. the map $\cdot x: \mathbb{Z}[x] \rightarrow \mathbb{Z}[x], v \mapsto v x$, is a locally nilpotent map, that is, $\mathbb{Z}[y]=\bigcup_{i \geq 1} \operatorname{ker}_{\mathbb{Z}[y]}\left(\cdot x^{i}\right)$. Therefore, the map $x-1$ is an isomorphism; hence $l=0$. This proves that the element $x-1$ is regular.

Let $A$ be a ring, $\sigma$ be its ring endomorphism, and $M$ be an $A$-module. The twisted by $\sigma A$-module ${ }^{\sigma} M$ is equal to $M$ as an abelian group, but the $A$-module structure on ${ }^{\sigma} M$ is given by the rule $a \cdot m=\sigma(a) m$, where $a \in A$ and $m \in M$.

Any right regular and right normal element $t$ of a ring $A$ determines the ring monomorphism $\tau_{t}: A \rightarrow A$ by the rule

$$
a t=t \tau_{t}(a) \text { for } a \in A \text {. }
$$

Clearly, $\tau_{t}(t)=t$, and so $\tau_{t}((t)) \subseteq(t)$ and we have the ring endomorphism $\overline{\tau_{t}}: \bar{A}:=$ $A /(t) \rightarrow \bar{A}$ given by the rule

$$
\overline{\tau_{t}}(a+(t))=\tau_{t}(a)+(t) .
$$

Proof of Theorem 1.3. Since the element $s$ is left regular (as $=0$ implies $a=0$ for $a \in A)$ and left normal $(A s=(s))$ there is the short exact sequence of $A$-modules

$$
0 \rightarrow A \stackrel{\cdot s}{\rightarrow} A \rightarrow \bar{A} \rightarrow 0 .
$$

This implies that $\operatorname{pd}_{A}(P) \leq 1$ for each projective $\bar{A}$-module $P$. In fact, $\operatorname{pd}_{A}(P)=1$ as the $A$-module $P$ cannot be projective since $s P=0$ and the element $s \in A$ is right regular. This proves the theorem when $n=0$. Let $n>0$ and use induction on $n$. Let $0 \rightarrow N \rightarrow P \rightarrow M \rightarrow 0$ be a short exact sequence of $\bar{A}$-modules, where $P$ is a projective $\bar{A}$-module. Then $N \neq 0$ and $\operatorname{pd}_{\bar{A}}(N)=n-1$. By induction $\operatorname{pd}_{A}(N)=n$ and $\operatorname{pd}_{A}(P)=1$. Therefore, $\operatorname{pd}_{A}(M) \leq n+1$ and $\operatorname{pd}_{A}(M)=n+1$ provided $n>1$.

If $n=1$, then $M=Q / R$ for some free $A$-module $Q$, where $R$ is its $A$-submodule such that $(s) Q \subseteq R$. Then the following short sequences of $\bar{A}$-modules are exact:

$$
\begin{gathered}
0 \rightarrow R /(s) Q \rightarrow Q /(s) Q \rightarrow M \rightarrow 0, \\
0 \rightarrow(s) Q /(s) R \rightarrow R /(s) R \rightarrow R /(s) Q \rightarrow 0 .
\end{gathered}
$$

By the assumption, $(s)=(t)=t A$ and $A t \subseteq t A$. Then $(s) Q /(s) R=t Q / t R$. Since the element $t \in A$ is right regular, the map

$$
t Q / t R \rightarrow Q / R, \quad t q+t R \mapsto q+R
$$

is a bijection. We claim that

$$
\operatorname{pd}_{\bar{A}}((s) Q /(s) R)=1 .
$$


Suppose that the first condition in the theorem holds. Since at $-t a \in\left(t^{2}\right)$ for all elements $a \in A$ and $(t) R=t R$, the map (13) is an $A$-module (and $\bar{A}$-module) isomorphism:

$$
a(t q+(t) R)=a t q+(t) R=t a q+(a t-t a) q+(t) R=t a q+(t) R
$$

as $(a t-t a) q \in(t)^{2} Q=(t)(s) Q \subseteq(t) R$ (since $(s) Q \subseteq R$ ). The claim is obvious in this case. Suppose that the second condition holds. Then there are obvious $\bar{A}$-module (and $A$-module) isomorphisms:

$$
(s) Q /(s) R=t Q / t R \simeq{ }^{\tau_{t}}(Q / R)={ }^{\tau_{t}} M \simeq{ }^{\overline{\tau_{t}}} M .
$$

Since $\overline{\tau_{t}}$ is an automorphism of the ring $\bar{A}, \operatorname{pd}_{\bar{A}}\left(\overline{\tau_{t}} M\right)=\operatorname{pd}_{\bar{A}}(M)=1$. The proof of the claim is complete. The $\bar{A}$-module $Q /(s) Q$ is projective and $\operatorname{pd}_{\bar{A}}(M)=1$; hence the $\bar{A}$-module $R /(s) Q$ is projective by (11). Then the short exact sequence (12) splits,

$$
R /(s) R \simeq(s) Q /(s) R \oplus R /(s) Q .
$$

Therefore, $\operatorname{pd}_{\bar{A}}(R /(s) R) \geq \operatorname{pd}_{\bar{A}}((s) Q /(s) R)=1$; hence $R$ is not a projective $A$ module (otherwise, we would have $\operatorname{pd}_{\bar{A}}(R /(s) R)=0$, a contradiction), and so $\operatorname{pd}_{A}(M)>1$ since $M=Q / R$ and $Q$ is a free $A$-module.

Theorem 1.3 is used in the proof of Lemma 2.4 (3), which is the key moment in the proof of Theorem 1.2 .

Lemma 2.4. Let $A$ be a ring. Then:

(1) For each $\mathbb{S}_{1}(A)$-module $M, \operatorname{pd}_{A}(M) \leq \operatorname{pd}_{\mathbb{S}_{1}(A)}(M)$.

(2) For each $A$-module $N, \operatorname{pd}_{\mathbb{S}_{1}(A)}\left(\mathbb{S}_{1}(A) \otimes_{A} N\right)=\operatorname{pd}_{A}(N)$.

(3) If $M$ is a nonzero $\mathbb{S}_{1}(A)$-module such that $(x-1) M=0$, then $\operatorname{pd}_{\mathbb{S}_{1}(A)}(M)=$ $\operatorname{pd}_{A}(M)+1$.

Proof. 1. Since $\mathbb{S}_{1}(A)$ is a free left $A$-module, any projective resolution of the $\mathbb{S}_{1}(A)$-module $M$ is also a projective resolution of the $A$-module $M$. Therefore, $\operatorname{pd}_{A}(M) \leq \operatorname{pd}_{\mathbb{S}_{1}(A)}(M)$.

2. It follows from the decomposition $\mathbb{S}_{1}(A)=\bigoplus_{i, j \in \mathbb{N}} x^{i} y^{j} A$ that the $A$-module $\mathbb{S}_{1}(A) \otimes_{A} N=\bigoplus_{i, j \in \mathbb{N}} A x^{i} y^{j} \otimes_{A} N \simeq\left({ }_{A} N\right)^{\left(\mathbb{N}^{2}\right)}$ is the direct sum of $\mathbb{N}^{2}$ copies of the $A$-module $N$. Then, by statement $1, \operatorname{pd}_{\mathbb{S}_{1}(A)}\left(\mathbb{S}_{1}(A) \otimes_{A} N\right) \geq \operatorname{pd}_{A}(N)$. If $P \rightarrow N$ is a projective resolution of the $A$-module $N$, then $\mathbb{S}_{1}(A) \otimes_{A} P \rightarrow \mathbb{S}_{1}(A) \otimes_{A} N$ is the projective resolution of the $\mathbb{S}_{1}(A)$-module $\mathbb{S}_{1}(A) \otimes_{A} N$ since $\mathbb{S}_{1}(A)_{A}$ is free. Therefore, $\operatorname{pd}_{\mathbb{S}_{1}(A)}\left(\mathbb{S}_{1}(A) \otimes_{A} N\right) \leq \operatorname{pd}_{A}(N)$.

3. Let $n=\operatorname{pd}_{A}(M)$. If $n=\infty$, then $\operatorname{pd}_{\mathbb{S}_{1}(A)}(M)=\infty$, by statement 1. If $n<\infty$, then we will see that the equality follows from Theorem 1.3 where $s=x-1$ and $t=y-1$. For, we have to check that the conditions of Theorem 1.3 hold for the elements $s$ and $t$ (we will see that both conditions 1 and 2 of Theorem 1.3 hold). By Lemma 2.3. the elements $s$ and $t$ of the ring $\mathbb{S}_{1}(A)$ are regular. By Lemma 2.1, the element $s$ is left normal and the element $t$ is right normal with $(s)=(t)$. Consider the inner derivation $\delta=$ ad $(t)=$ ad $(y)$ of the ring $\mathbb{S}_{1}(A)$ (recall that ad $(t)(a)=t a-a t$ for all elements $\left.a \in \mathbb{S}_{n}(A)\right)$. Since $\delta(A)=0, \delta(y)=0$ and $\delta(x)=y x-x y=E_{00}$, we see that $\delta\left(\mathbb{S}_{1}(A)\right) \subseteq F(A)$. Notice that $F(A)^{2}=F(A)$, by (21). By Lemma 2.1, $F(A) \subseteq(y-1)$. Then $a t-t a \in F(A)=F(A)^{2} \subseteq(y-1)^{2}=\left(t^{2}\right)$ for all elements $a \in \mathbb{S}_{1}(A)$. By Lemma $2.1(2), \overline{\mathbb{S}_{n}(A)}=\mathbb{S}_{n}(A) /(t)=A$ and the ring endomorphism $\overline{\tau_{t}}$ is the identity automorphism of the ring $A$. This means that the 
assumptions of Theorem 1.3 hold for the elements $s=x-1$ and $t=y-1$, and so $\operatorname{pd}_{\mathbb{S}_{1}(A)}(M)=n+1$ by Theorem 1.3 .

Proof of Theorem 1.2 . Since $\mathbb{S}_{n}(A)=\mathbb{S}_{1}\left(\mathbb{S}_{n-1}(A)\right)$, it suffices to prove the statements when $n=1$. In view of the involution $*$ on the algebra $\mathbb{S}_{1}(\mathbb{Z})$ such that $y^{*}=$ $x$, and $\mathbb{S}_{1}(A)=A \otimes_{\mathbb{Z}} \mathbb{S}_{1}(\mathbb{Z})$, it suffices to show that $\lg \operatorname{gldim}\left(\mathbb{S}_{1}(A)\right)=\lg \operatorname{gldim}(A)+1$.

By Lemma 2.4 (2), l.gldim $\left(\mathbb{S}_{1}(A)\right) \geq \operatorname{lggldim}(A)$, and so the equality is true if $\operatorname{l.g} \operatorname{ldim}(A)=\infty$. Suppose that $\lg \operatorname{gldim}(A)<\infty$. By Lemma 2.4 $(3), \lg \operatorname{gldim}\left(\mathbb{S}_{1}(A)\right)$ $\geq \lg \operatorname{gldim}(A)+1$ since each $A$-module $N$ can be seen as the $\mathbb{S}_{1}(A)$-module which is annihilated by the element $x-1$ since $\left.{ }_{A} \mathbb{S}_{1}(A)\right)_{A}=A \oplus(x-1)$ and $(x-1)=$ $\mathbb{S}_{1}(A)(x-1)$. It remains to show that $\operatorname{pd}_{\mathbb{S}_{1}(A)}(M) \leq \lg \operatorname{gldim}(A)+1$ for all nonzero $\mathbb{S}_{1}(A)$-modules $M$. There is the short exact sequence of $\mathbb{S}_{1}(A)$-modules (by Lemma 2.3 and (8) )

$$
0 \rightarrow \mathbb{S}_{1}(A) \otimes_{A} M \stackrel{\cdot(x-1)}{\rightarrow} \mathbb{S}_{1}(A) \otimes_{A} M \rightarrow M \rightarrow 0 .
$$

Notice that $\mathbb{S}_{1}(A) \otimes_{A} M=\mathbb{S}_{1}(\mathbb{Z}) \otimes_{\mathbb{Z}} A \otimes_{A} M \simeq \mathbb{S}_{1}(\mathbb{Z}) \otimes_{\mathbb{Z}} M$, and the map $\cdot(x-1)$ above is equal to the map

$$
\cdot(x-1) \otimes \operatorname{id}_{M}: \mathbb{S}_{1}(\mathbb{Z}) \otimes_{\mathbb{Z}} M \rightarrow \mathbb{S}_{1}(\mathbb{Z}) \otimes_{\mathbb{Z}} M .
$$

By Lemma 2.4 $\operatorname{pd}_{\mathbb{S}_{1}(A)}\left(\mathbb{S}_{1}(A) \otimes_{A} M\right)=\operatorname{pd}_{A}(M) \leq \operatorname{lggldim}(A)$. By (14), $\operatorname{pd}_{\mathbb{S}_{1}(A)}(M) \leq \operatorname{lggldim}(A)+1$. The proof of the theorem is complete.

Corollary 2.5. (1) [6] Let $K$ be a field. Then $\lg \operatorname{ldim}\left(\mathbb{S}_{n}(K)\right)=\operatorname{r.gldim}\left(\mathbb{S}_{n}(K)\right)$ $=n$.

(2) $\operatorname{l.gldim}\left(\mathbb{S}_{n}(\mathbb{Z})\right)=\operatorname{r.gldim}\left(\mathbb{S}_{n}(\mathbb{Z})\right)=n+1$.

Theorem 2.6. Let $A$ be an algebra over a field $K$ such that either w.dim $(A)=$ $\operatorname{l.g} \operatorname{ldim}(A)$ (e.g., $A$ is a left Noetherian algebra) or w.dim $(A)=\operatorname{r.gldim}(A)(e . g ., A$ is a right Noetherian algebra). Then $\mathrm{w} \cdot \operatorname{dim}\left(\mathbb{S}_{n}(A)\right)=\mathrm{w} \cdot \operatorname{dim}(A)+n$.

Proof. For any two $K$-algebras $R$ and $S$, w.dim $(R \otimes S) \geq$ w.dim $(R)+$ w.dim $(S)$ (Theorem 16, [1]). Therefore, w.dim $\left(\mathbb{S}_{n}(A)\right)=\mathrm{w} \cdot \operatorname{dim}\left(\mathbb{S}_{n} \otimes A\right) \geq \mathrm{w} \cdot \operatorname{dim}\left(\mathbb{S}_{n}\right)+$ $\mathrm{w} \cdot \operatorname{dim}(A)=n+\mathrm{w} \cdot \operatorname{dim}(A)$ since $\mathrm{w} \cdot \operatorname{dim}\left(\mathbb{S}_{n}\right)=n$, Corollary 6.8, [6]. The inverse inequality follows from Theorem 1.2 and the fact that $\mathrm{w} \cdot \operatorname{dim}(R) \leq$ $\min \{\lg \operatorname{gldim}(R), \mathrm{r} \cdot \operatorname{gldim}(R)\}$ for all rings $R$. Let $d=1$.gldim (resp. $d=$ r.gldim). If $A$ is a left (resp. right) Noetherian algebra, then $d(A)=$ w.dim $(A)$ and

$$
\mathrm{w} \cdot \operatorname{dim}\left(\mathbb{S}_{n}(A)\right) \leq d\left(\mathbb{S}_{n}(A)\right)=d(A)+n=\mathrm{w} \cdot \operatorname{dim}(A)+n .
$$

The proof of the theorem is complete.

Corollary 2.7. w.dim $\left(\mathbb{S}_{n}(\mathbb{Z})\right)=n+1$.

In his report the referee asked the following two questions ( $K$ is a field):

Q1. Is the algebra $\mathbb{S}_{n}(K)$ coherent?

Q2. What is $K_{0}\left(\mathbb{S}_{n}(K)\right)$ ?

Theorem 2.8 gives the answer to the first question. A module $M$ over a ring $R$ is finitely presented if there is an exact sequence of modules $R^{m} \rightarrow R^{n} \rightarrow M \rightarrow 0$. A finitely generated module is a coherent module if every finitely generated submodule is finitely presented. A ring $R$ is a left (resp. right) coherent ring if the module ${ }_{R} R$ (resp. $R_{R}$ ) is coherent. $A$ ring $R$ is a left coherent ring iff, for each element $r \in R, \operatorname{ker}_{R}(\cdot r)$ is a finitely generated left $R$-module and the intersection of two 
finitely generated left ideals is finitely generated [21, Proposition 13.3]. Each left Noetherian ring is left coherent but not vice versa.

Theorem 2.8. Let $K$ be a field and $\mathbb{S}_{n}=\mathbb{S}_{n}(K)$. Then the algebra $\mathbb{S}_{n}$ is a left coherent algebra iff the algebra $\mathbb{S}_{n}$ is a right coherent algebra iff $n=1$.

Proof. In view of existence of the involution $*$ on the algebra $\mathbb{S}_{n}$ the first 'iff' is obvious since the algebra $\mathbb{S}_{n}$ is self-dual; i.e. it is isomorphic to its opposite algebra $\mathbb{S}_{n}^{o p}\left(\right.$ via $\mathbb{S}_{n} \rightarrow \mathbb{S}_{n}^{o p}, a \mapsto a^{*}$ ). By Corollary $2.5(1)$, the algebra $\mathbb{S}_{1}$ is a hereditary algebra hence coherent.

If $n \geq 2$, then the algebra $\mathbb{S}_{n}$ is not left coherent as the left $\mathbb{S}_{n}$-module $\mathcal{K}:=$ $\operatorname{ker}_{\mathbb{S}_{n}}\left(\cdot\left(x_{1}-x_{2}\right)\right)$ is not finitely generated. In more detail, $\mathcal{K}=\mathcal{K}^{\prime} \otimes \mathbb{S}_{n-2}$, where $\mathcal{K}^{\prime}:=\operatorname{ker}_{\mathbb{S}_{2}}\left(\cdot\left(x_{1}-x_{2}\right)\right)$ and $\mathbb{S}_{n}=\mathbb{S}_{2} \otimes \mathbb{S}_{n-2}$. It suffices to show that the $\mathbb{S}_{2}$-module $\mathcal{K}^{\prime}$ is an infinite direct sum of nonzero $\mathbb{S}_{2}$-modules (then $\mathcal{K}$ is an infinite direct sum of nonzero $\mathbb{S}_{n}$-modules; hence $\mathcal{K}$ is not a finitely generated $\mathbb{S}_{n}$-module and as a result the algebra $\mathbb{S}_{n}$ is not left coherent). So, we can assume that $n=2$. Recall that $\mathbb{S}_{2}=\mathbb{S}_{1}(1) \otimes \mathbb{S}_{1}(2)$, where $\mathbb{S}_{1}(k)=K\left\langle x_{k}, y_{k}\right\rangle \simeq \mathbb{S}_{1}$ for $k=1,2 ; F(k):=$ $\bigoplus_{i, j \in \mathbb{N}} K E_{i j}(k)$ is the only proper ideal of the algebra $\mathbb{S}_{1}(k)$ and $\mathbb{S}_{1}(k) / F(k)=$ $L_{1}(k):=K\left[x_{k}, x_{k}^{-1}\right]$. The ideal $\mathfrak{a}_{2}:=F(1) \otimes \mathbb{S}_{1}(2)+\mathbb{S}_{1}(1) \otimes F(2)$ of the algebra $\mathbb{S}_{1}$ is such that $\mathbb{S}_{2} / \mathfrak{a}_{2} \simeq L_{1}(1) \otimes L_{1}(2)=K\left[x_{1}, x_{1}^{-1}, x_{2}, x_{2}^{-1}\right]$ is a commutative domain. Therefore, $\mathcal{K}=\operatorname{ker}_{\mathfrak{a}_{2}}\left(\cdot\left(x_{1}-x_{2}\right)\right)$ since the element $x_{1}-x_{2}$ is a nonzero element of the domain $L_{1}(1) \otimes L_{1}(2)$. It follows from the short exact sequence of right $\mathbb{S}_{2}$-modules

$$
0 \rightarrow F:=F(1) \otimes F(2) \rightarrow \mathfrak{a}_{2} \rightarrow \mathfrak{a}_{2} /\left(F(1) \otimes \mathbb{S}_{1}(2)\right) \simeq L_{1}(1) \otimes \mathbb{S}_{1}(2) \rightarrow 0
$$

and the equality $\operatorname{ker}_{L_{1}(1) \otimes \mathbb{S}_{1}(2)}\left(\cdot\left(x_{1}-x_{2}\right)\right)=0$ (use the fact that the algebra $L_{1}(1)=$ $\bigoplus_{i \in \mathbb{Z}} K x_{1}^{i}$ is $\mathbb{Z}$-graded $)$ that $\mathcal{K}=\operatorname{ker}_{F}\left(\cdot\left(x_{1}-x_{2}\right)\right)$. The ideal

$$
F=\bigoplus_{\alpha, \beta \in \mathbb{N}^{2}} K E_{\alpha \beta}=\bigoplus_{\beta \in \mathbb{N}^{2}} \mathbb{S}_{2} E_{0 \beta}=\bigoplus_{\alpha \in \mathbb{N}^{2}} E_{\alpha 0} \mathbb{S}_{2}
$$

is the direct sum of nonzero left ideals $\mathbb{S}_{2} E_{0 \beta}=\bigoplus_{\alpha \in \mathbb{N}^{2}} K E_{\alpha \beta}$ and the direct sum of nonzero right ideals $E_{\alpha 0} \mathbb{S}_{2}=\bigoplus_{\beta \in \mathbb{N}^{2}} K E_{\alpha \beta}$. Using the identities

$$
E_{i, j}(k) x_{k}= \begin{cases}E_{i, j-1}(k) & \text { if } j \geq 1, \\ 0 & \text { if } j=0,\end{cases}
$$

we see that $\operatorname{ker}_{E_{\alpha 0} \mathbb{S}_{2}}\left(\cdot\left(x_{1}-x_{2}\right)\right)=\bigoplus_{s \in \mathbb{N}} K v_{\alpha}(s)$, where $v_{\alpha}(s):=\sum_{\beta_{1}+\beta_{2}=s} E_{\alpha \beta}$ and $\beta=\left(\beta_{1}, \beta_{2}\right) \in \mathbb{N}^{2}$. Notice that $v_{\alpha}(s)=x^{\alpha} v_{0}(s)$ for all $\alpha \in \mathbb{N}^{2}$ and $y^{\beta} v_{0}(s)=0$ for all $\beta \in \mathbb{N}^{2} \backslash\{0\}$. Therefore,

$$
\operatorname{ker}_{F}\left(\cdot\left(x_{1}-x_{2}\right)\right)=\bigoplus_{\alpha \in \mathbb{N}^{2}} \operatorname{ker}_{E_{\alpha 0} \mathbb{S}_{2}}\left(\cdot\left(x_{1}-x_{2}\right)\right)=\bigoplus_{\alpha \in \mathbb{N}^{2}, s \in \mathbb{N}} K v_{\alpha}(s)=\bigoplus_{s \in \mathbb{N}} \mathbb{S}_{2} v_{0}(s)
$$

is an infinite direct sum of nonzero left ideals of the algebra $\mathbb{S}_{2}$, as required.

\section{ACKNOWLEDGEMENT}

The author would like to thank the referee for the interesting comments and challenging questions. 


\section{REFERENCES}

[1] M. Auslander, On the dimension of modules and algebras (3). Global dimension, Nagoya Math. J. 9 (1955) 67-77. MR0074406(17:579a)

[2] R. Baer, Inverses and zero-divisors, Bull. Amer. Math. Soc. 48 (1942) 630-638. MR0006997 (4:70a)

[3] H. Bass, Algebraic K-theory. W. A. Benjamin, Inc., New York-Amsterdam, 1968. MR0249491 $(40: 2736)$

[4] V. V. Bavula, Global dimension of generalized Weyl algebras. Representation theory of algebras (Cocoyoc, 1994), 81-107, CMS Conf. Proc., 18, Amer. Math. Soc., Providence, RI, 1996. MR $1388045(97 \mathrm{e}: 16018)$

[5] V. V. Bavula, Tensor homological minimal algebras, global dimension of the tensor product of algebras and of generalized Weyl algebras. Bull. Sci. Math. 120 (1996) no. 3, 293-335. MR.1399845 (97f:16015)

[6] V. V. Bavula, The algebra of one-sided inverses of a polynomial algebra, Journal Pure Appl. Algebra 214 (2010) 1874-1897. MR2608115 (2011f:16069)

[7] V. V. Bavula, The group of automorphisms of the algebra of one-sided inverses of a polynomial algebra, arXiv:math.AG/0903.3049.

[8] V. V. Bavula, $\mathrm{K}_{1}\left(\mathbb{S}_{1}\right)$ and the group of automorphisms of the algebra $\mathbb{S}_{2}$ of one-sided inverses of a polynomial algebra in two variables, Journal of K-theory and its Appl. to Algebra, Geometry, Analysis and Topology, to appear, arXiv:0906.0600.

[9] V. V. Bavula, The group of automorphisms of the algebra of one-sided inverses of a polynomial algebra, II. arXiv:math.AG:0906.3733.

[10] V. V. Bavula, The group $\mathrm{K}_{1}\left(\mathbb{S}_{n}\right)$ of the algebra of one-sided inverses of a polynomial algebra, arXiv:math.KT:1005.3550.

[11] J.-E. Bjork, The global homological dimension of some algebras of differential operators, Invent. Math. 17 (1972) 67-78. MR0320078 (47:8619)

[12] K. A. Brown, C. R. Hajarnavis and A. B. MacEacharn, Noetherian rings of finite global dimension. Proc. London Math. Soc. 44 (1982) 349-71. MR647437(84a:16025)

[13] S. Eilenberg, A. Rosenberg and D. Zelinsky, On the dimension of modules and algebras, VIII. Dimension of tensor products, Nagoya Math. J. 12 (1957) 71-93. MR0098774 (20:5229)

[14] L. Gerritzen, Modules over the algebra of the noncommutative equation $y x=1$. Arch. Math. (Basel) 75 (2000) no. 2, 98-112. MR1767169(2001c:16013)

[15] K. R. Goodearl, Global dimension of differential operator rings. II, Trans. Amer. Math. Soc. 209 (1975) 65-85. MR0382359 (52:3244)

[16] N. Jacobson, Some remarks on one-sided inverses, Proc. Amer. Math. Soc. 1 (1950) 352-355. MR0036223 (12:75e)

[17] N. Jacobson, "Structure of rings", Amer. Math. Soc. Colloq., Vol. XXXVII (rev. ed.), Amer. Math. Soc., Providence, RI, 1968. MR0222106 (36:5158)

[18] I. Kaplansky, Fields and Rings, Chicago Lectures in Mathematics. University of Chicago Press, Chicago-London, 1969. MR0269449 (42:4345)

[19] G. S. Rinehart and A. Rosenberg, The global dimensions of Ore extensions and Weyl algebras. Algebra, topology, and category theory (a collection of papers in honor of Samuel Eilenberg), pp. 169-180. Academic Press, New York, 1976. MR0409563 (53:13317)

[20] A. Rosenberg and J. T. Stafford, Global dimension of Ore extensions. Algebra, topology, and category theory (a collection of papers in honor of Samuel Eilenberg), pp. 181-188. Academic Press, New York, 1976. MR0409564 (53:13318)

[21] B. Stenström, Rings of quotients. An introduction to methods of ring theory. Springer-Verlag, New York-Heidelberg, 1975. MR0389953 (52:10782)

Department of Pure Mathematics, University of Sheffield, Hicks Building, Sheffield S3 7RH, United Kingdom

E-mail address: v.bavula@sheffield.ac.uk 